\title{
Intermédialités
}

Histoire et théorie des arts, des lettres et des techniques

Intermediality

History and Theory of the Arts, Literature and Technologies

\section{Questions et hypothèses à partir des systèmes d'écritures : remédiation ou plurimédialité?}

\section{Philippe Despoix}

Numéro 6, automne 2005

Remédier

Remediation

URI : https://id.erudit.org/iderudit/1005508ar

DOI : https://doi.org/10.7202/1005508ar

Aller au sommaire du numéro

Éditeur(s)

Centre de recherche sur l'intermédialité

ISSN

1705-8546 (imprimé)

1920-3136 (numérique)

Découvrir la revue

Citer cet article

Despoix, P. (2005). Questions et hypothèses à partir des systèmes d'écritures : remédiation ou plurimédialité? Intermédialités / Intermediality, (6), 95-106.

https://doi.org/10.7202/1005508ar
Résumé de l'article

Le concept de "remédiation » a été jusqu'ici essentiellement exploré à partir des arts visuels et électroniques. Cette contribution vise à mettre en perspective historique et comparée l'idée de remédiation continue dans laquelle s'inscrirait chaque nouveau médium. En prenant pour champ d'enquête les types les plus divers de systèmes d'écriture (graphisme spatial, écritures linéarisées, systèmes idéographiques, syllabaires, alphabets, etc.), elle essaie de proposer quelques hypothèses concernant les figures selon lesquelles l'écrit peut « remédier » — ou pas — (à) la parole. 


\title{
Questions et hypothèses à partir des systèmes d'écritures: remédiation ou plurimédialité?
}

\author{
Philippe Despoix

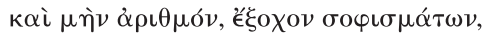

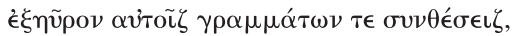 \\ $\mu \nu \eta \dot{\eta} \mu \eta \nu \dot{\alpha} \pi \alpha \nu \tau \omega \nu, \mu о v \sigma o \mu \eta \tau о \rho ' ~ ' ́ \rho \gamma \alpha \tau \iota \nu^{1}$. \\ (Eschyle, Prométhée enchaîné, 459-61)
}

\begin{abstract}
[ concept de "remédiation » a été jusqu'ici essentiellement exploré à partir du domaine des arts visuels et électroniques. Sans qu'elle soit explicitée, l'idée en était néanmoins déjà fortement esquissée dans la thèse macluhanienne selon laquelle le contenu de tout médium serait un autre médium plus ancien. On se souvient de la formule: "The content of writing is speech, just as the written word is the content of print, and print is the content of the telegraph ${ }^{2}$.» Tout à la fois abrupte et productive, elle mettait d'emblée l'accent sur la chaîne de remédiation continue dans laquelle s'inscrirait tout nouveau médium. À «l'origine» d'une telle chaîne, il y aurait l'écriture en tant que remédiation de la parole. L'affirmation résiste-t-elle à un parcours comparé à travers les principaux types de cultures écrites? Sans doute, la formule se réfère-t-elle implicitement à l'écriture alphabétique et en assume le caractère spécifiquement «occidental». Aussi, sa mise en perspective par les systèmes d'écriture dans leur diversité même (graphisme spatial, écritures linéarisées, systèmes idéographiques ou phonétisés, etc.) constitue-t-elle un bon terrain pour apprécier la valeur opératoire d'un tel concept. Essayons de formuler ici un certain nombre de questions et hypothèses qui pourraient guider un chantier de recherche.
\end{abstract}

1. «Ce fut celle du nombre, la première de toutes les sciences, que j'inventai pour eux, ainsi que l'assemblage des lettres, mémoire de toutes choses, labeur qui enfante les arts.»

2. Marshall McLuhan, Understanding Media: The Extensions of Man, $2^{\mathrm{e}}$ éd., New York, Scaborough, London, McGraw-Hill, 1964, p. 23 sq. 
Qu'est-ce qu'écrire? Comment définir, tout d'abord, l'écriture? Pour l'appréhender en tant que médium, n’est-il pas en premier lieu nécessaire de la décrire indépendamment de tout système de signification, dans sa dimension a priori de technique graphique? Elle se distingue alors directement des autres types d'activité symbolique (gestuelles ou verbales) par ses traces matérielles durables. On peut interroger l'existence même de telles traces et considérer l'écriture comme une activité, une technique du corps (en général de la main, en liaison avec la vue ou l'ouïe) appliquée à un outil d'inscription sur un support3. Cette prémisse méthodologique permet d'en envisager les divers types, historiquement et comparativement, en tant que médium technique et sous l'angle d'une continuité physique des formes d'activité graphique face aux très diverses fonctions qu'elle peut remplir.

Toute trace graphique organisée est-elle en ce sens une écriture? Répondre par l'affirmative signifie faire commencer cette dernière à la "préhistoire », par le dessin rupestre. La paléontologie a montré que la naissance du graphisme impliquait chez l'homme un rapport nouveau entre les pôles fonctionnels mainoutil et face-parole dans lequel la vue prend une place dominante. On sait que les premières traces graphiques humaines renvoient à une abstraction poussée (rythmes, symboles de la différence des sexes) puis, massivement, à la représentation animale. Il s'agit toutefois d'un graphisme purement organisé dans l'espace de la roche au sein duquel aucune dimension temporelle, aucun thème narratif n'est déchiffrablet. L'hypothèse, plausible, d'un cadre rituel n’est pas vérifiable. Il ne semble pas exister dans la facture même de ce que l'on a appelé «mythogrammes» de renvoi interne à des séquences strictement linguistiques. Ces premiers types d'écritures obéiraient à un graphisme autonome, purement spatial.

\section{II}

Dans quel contexte apparaissent les écritures linéarisées, c'est-à-dire pour lesquelles l'espace graphique est asservi à un déroulement temporel? Les protoécritures linéaires, qui scellent l'entrée de ce que nous appelons «histoire», se caractérisent par une sérialisation de symboles référant à une action extérieure.

3. Voir Marcel Mauss, «Les techniques du corps », dans Sociologie et anthropologie, Paris, Presses universitaires de France, coll. "Quadrige», 1985 [1950], p. 365 sq.

4. Voir André Leroi-Gourhan, Le geste et la parole, tome 2, Paris, Albin Michel, 1964 , p. $241 s q$. 


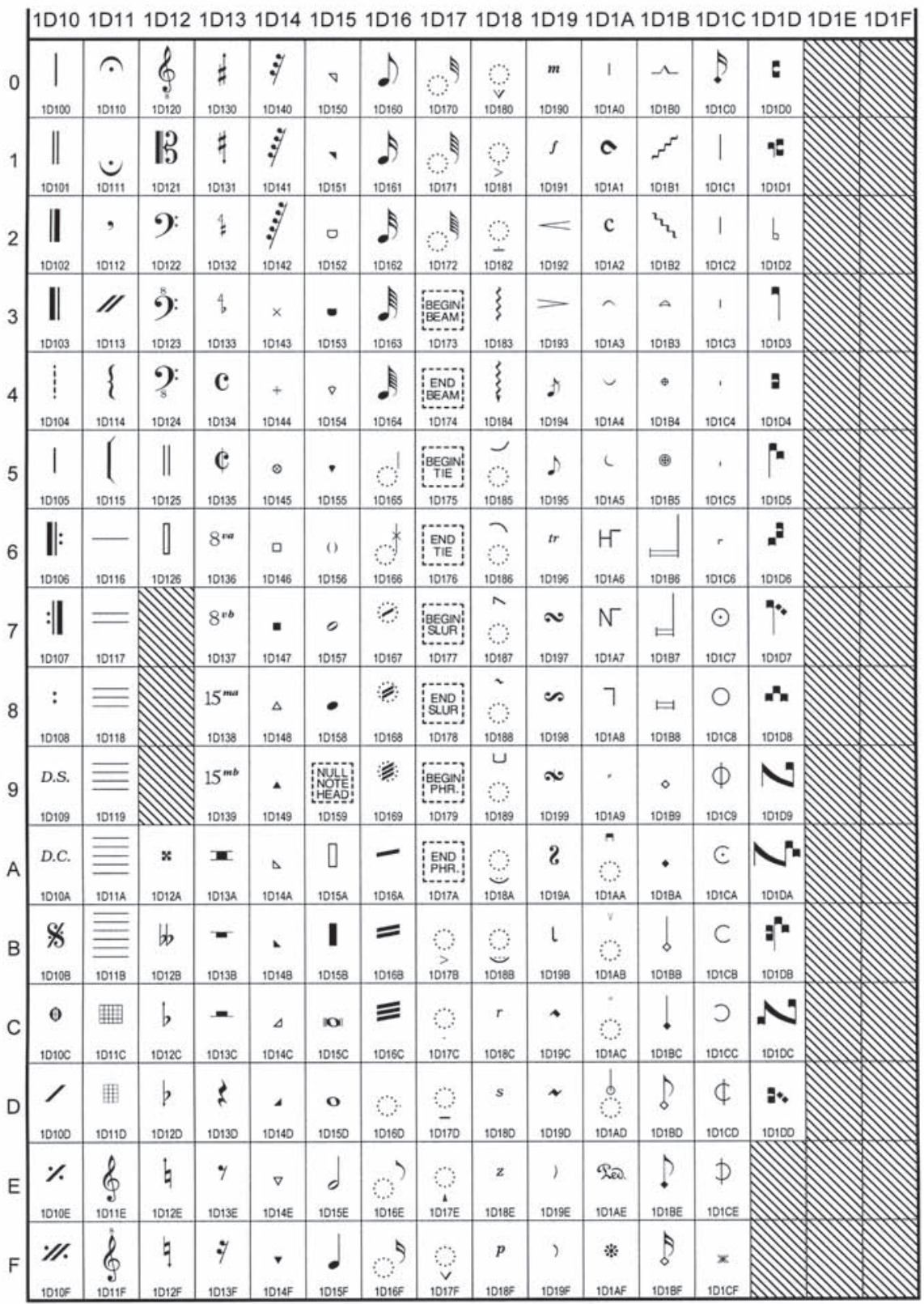


Il est frappant qu'elles fixent d'abord des échanges, des transferts de biens. Selon les hypothèses les plus partagées, ce sont des formes de comptabilité qui auraient présidé à la naissance des premiers systèmes complexes ${ }^{5}$ : écriture de choses qui enregistre avant tout la trace de transactions matérielles. La possibilité de noter des échanges proprement linguistiques par le moyen de l'écrit a été probablement découverte dans ce cadre, «par hasard», en enregistrant des quantités.

La numération constituerait-elle le seuil décisif de linéarisation de l'activité graphique, condition d'un développement «logographique »? L'archéologie de la culture élamite suggère en effet que forme de numération écrite, écriture et monnaie naissent en bloc des techniques de comptabilité matérielle ${ }^{6}$. C'est d'abord dans les contraintes des formes (probablement ritualisées) d'échanges réguliers que les protosystèmes d'écriture commencent à signifier une action? Il est décisif que les opérations de numération et de nomination apparaissent ici comme les vecteurs concomitants entraînant la représentation écrite. Ces premières écritures qui nomment et décomptent les choses (animaux d'élevage et plantes cultivées essentiellement) fixent et formalisent de manière symbolique un échange social, mais elles autorisent de plus un premier lien entre un objet, sa représentation picturale abstraite et sa désignation par la parole.

\section{III}

L'histoire des écritures nous apprend que l'évolution proprement logographique peut avoir lieu dans deux directions types: par abstraction de la représentation de choses vers une idéographie qui vient redoubler chaque mot d'un signe graphique singulier; ou encore par développement d'un système phonographique qui note directement les sons de la parole. Peut-on dire que les écritures idéographiques et phonétiques constitueraient des formes, certes distinctes, de remédiation de la langue par l'écriture? Linguistiquement, l'idéogramme est un signifiant matérialisé au même titre que le mot articulé, alors que le signe phonétique ne constitue qu’une représentation dérivée du signifiant verbal, sa «reproduction » graphique.

5. À ce propos, voir Jean Bottéro, «La première écriture», dans Jean Bottéro, Clarisse Herrenschmidt, Jean-Pierre Vernant, L'Orient ancien et nous: l'écriture, la raison, les dieux, Paris, Albin Michel, coll. «Chaire de l'IMA», 1996, p. 38.

6. Voir Clarisse Herrenschmidt, «La civilisation élamite et l'écriture», dans Jean Bottéro et al., L'Orient ancien et nous, p. 98 sq.

7. Sur la signification rituelle de l'écriture voir aussi Jan Assmann, Das kulturelle Gedächtnis. Schrift, Erinnerung und politische Identität in frühen Hochkulturen, Munich, Beck, 1992. 
Même si les deux types d'écritures sont en réalité rarement exclusifs et forment le plus souvent des systèmes composites, l'un des aspects domine toujours assez nettement pour en rendre la différence significative.

Ne serait-il pas fondamental de résister ici au caractère d'évidence - pour nous occidentaux - de l'interaction «obligée » entre le médium de la parole et celui de l'écrit ${ }^{8}$ ? Si les systèmes d'écritures à tendance idéographique redoublent littéralement (mot par mot) de manière graphique les compétences du langage, n'y aurait-il pas lieu de parler dans ce cas de plurimédialité des instruments de la parole et de l'écriture? Alors que dans un système phonétisé, la poésie, même écrite, peut être complètement oralisée, il existe dans la calligraphie de la poésie chinoise un élément graphique qui esthétiquement reste complètement irréductible à toute transposition verbale. Ici le rapport de parallélisme distant conservé entre ces deux instances du langage, parlé et écrit, contraste fortement avec celui de remédiation de la parole effectivement réalisé par les écritures phonétiques.

En d'autre termes: comment sortir du dilemme «eurocentriste » qui a trop souvent consisté à faire de l'idéographie l'autre de l'alphabet? Une voie probable consisterait à « ethnographier» de manière comparée les conséquences culturelles de ces systèmes hétérogènes d'inscription: analyser, à partir des techniques d'écriture elles-mêmes, les modes d'apprentissage d'aperception et de mémorisation du langage qui privilégient de manière différenciée la vision ou l'audition; en explorer les effets sur la configuration du rapport entre spatialité et temporalité; en spécifier les diverses dynamiques induites entre la parole et l'écriture, de même que les répercussions cognitives, politiques, esthétiques, etc. qui peuvent leur être attachées. Il s'agirait, à partir de telles enquêtes, de construire la distinction "plurimédialité - remédiation » comme un rapport typologique et non d'opposition ou de dépassement historique.

\section{IV}

Ces questions demandent à être précisées du côté même des systèmes phonétisés: quelles sont les différentes possibilités de formalisation de la phonation par une écriture? Si avec les écritures phonétiques on peut parler de remédiation de la parole au sens propre, les modes d'analyse phonologique effectués distinctement par chacun des systèmes types prêtent-ils à conséquence? Le premier connu est le syllabaire, point d'aboutissement de l'écriture cunéiforme. Les succès de sa

8. Voir entre autres Jack Goody, The Interface Between the Written and the Oral, Cambridge, Cambridge University Press, 1987 et Walter Ong, Orality and Literacy: The Technologizing of the Word, London, Methuem, 1982. 
diffusion sont vraisemblablement dus à ce qu'il est fondé sur le caractère « universel » de la syllabe comme unité d'articulation linguistique. Apparues en contexte multilingue, les écritures syllabiques permettent de retranscrire à l'aide d'un seul ensemble de signes plusieurs langues différentes. Elles autorisent une réduction radicale du nombre de caractères utilisés: en tendant à un signe graphique par syllabe, elles allègent considérablement la mémoire visuelle du scripteur. Elles réalisent une véritable synchronisation de la main inscrivant les signes dans l'espace linéarisé des tablettes et de la parole telle qu'articulée dans le temps.

Mais qu'en est-il des autres modes de phonétisation? On sait que les syllabaires ont d'abord évolué vers des systèmes graphiques consonantiques. Ces écritures (dont relève à l'origine la notation du phénicien, de l'araméen, de l'hébreu et, indirectement, de l'arabe) procèdent par diminution du nombre de signes nécessaires à reproduire la langue parlée en transcrivant les syllabes à l'aide de leurs seules consonnes communes. Cette simplification ramène le nombre de caractères à moins de trente mais implique une perte dans l'univocité de l'écrit. Ce n'est plus la syllabe entendue qui sert d'unité graphique, mais la syllabe telle que déchiffrée (reconstituée et articulée) par le sujet lisant 9 . Celui-ci doit parfaitement connaître la langue pour pouvoir lire. Le choix de noter seulement les discontinuités de l'articulation (sans ses variations de timbre) crée un nouveau déséquilibre entre voix et écriture. La remédiation de la parole n’y est plus «transparente » et s'y trouve soumise à l'interprétation. En obligeant le lecteur à reconstituer l'articulation verbale, les écritures consonantiques maintiennent une transcendance de la parole par rapport à l'écrit ${ }^{10}$. Celui-ci reste ainsi dépendant d'une oralisation complémentaire, fait d'écriture qui a particulièrement été mis en valeur par les religions monothéistes.

L'écriture proprement alphabétique, apport singulier de la culture grecque, pousse plus loin encore l'analyse du langage parlé. Constitue-t-elle pour autant une rationalisation ultime? En introduisant la graphie des voyelles, elle reprend le principe de réduction radicale du nombre de caractères propre aux écritures consonantiques tout en maintenant l'univocité parole-écriture atteinte par les syllabaires. C'est un autre type d'analyse phonologique de la langue parlée que l'alphabet propose, non plus basée sur l'opposition des syllabes entre elles mais en premier lieu sur celle des consonnes et des voyelles. L'écriture alphabétique

9. Voir Clarisse Herrenschmidt, «Alphabets consonantiques, alphabets grecs, cunéiforme, vieux perse », dans Jean Bottéro et al., L'Orient ancien et nous, p. 124 sq.

10. Voir Clarisse Herrenschmidt, «L'écriture et quelques questions juives et grecques », dans Jean Bottéro et al., L'Orient ancien et nous, p. 164. 
note de fait toutes les positions de l'appareil phonatoire, elle rend explicite - pour la première fois - l'analyse de la parole comme technique du corps. Il ne s'agit plus d'une découpe du matériau sonore tel qu'entendu, mais tel que produit par les différents points d'appui constitutifs de l'organe de la parole. C'est l'analyse acoustique de cette production verbale que l'alphabet visualise de manière graphique. Cependant, le système d'opposition consonnes-voyelles n'est pas sans ambiguïté car «asymétrique». Contrairement aux voyelles, les consonnes (en particulier les occlusives) ne sont pas des sons au sens propre du terme, mais plutôt des attaques ou des arrêts sonores. La rationalisation qu'effectue l'alphabet grec bute de fait sur une irrationalité plus profonde, car la simplification de la lecture y est atteinte au détriment de l'équilibre: un signe = un son (problème qui s'est, entre autres, traduit par l'élimination de la graphie du souffle, le $h$ aspiré $)^{11}$. Néanmoins, en tant que forme de remédiation univoque et facile à maîtriser de la parole, l'écriture alphabétique peut elle-même devenir l'une de ses formes privilégiée.

La portée des différences entre les techniques scripturaires amène à s'interroger sur les modes de valorisation des rapports possibles entre l'oral et l'écrit. Dans la structuration des schèmes cognitifs par chaque type d'écriture, c'est à la fois la configuration réciproque de l'espace et de la temporalité et celle des formes de mémorisation qui se noue. Là encore, la comparaison entre des cultures à écriture phonétisée et celles ancrées dans l'idéographie est éclairante. La formation du lettré chinois s'adressant autant, si ce n'est plus, à l'œil qu'à l'oreille, c'est spécifiquement l'écriture et la lecture des productions littéraires qui ont été valorisées dans cette tradition pour laquelle la forme épique orale a longtemps été considérée comme vulgaire ${ }^{12}$. De manière opposée, la culture brahmanique de l'Inde offre l'exemple singulier d'une tradition écrite phonétisée qui insiste sur

11. Clarisse Herrenschmidt, «L'écriture et quelques questions juives et grecques», dans Jean Bottéro et al., L'Orient ancien et nous, p. 175 sq. ; voir également Eric Havelock, Aux origines de la civilisation écrite, trad. Escobar Moreno, Paris, Éditions François Maspero, 1981 [1976].

12. Voir Max Weber, Die Wirtschaftsethik der Weltreligionen I. Konfuzianismus und Taoismus. Schriften 1915-1920 (MWS I/19), Tübingen, J.C.B. Mohr Siebeck, 1991, p. 122 sq. (Confucianisme et taoïsme, trad. Catherine Colliot-Thélène et Jean-Pierre Grossein, Paris, Éditions Gallimard, coll. «Bibliothèque des sciences humaines», 2000, p. 182 sq.) 
la transmission exclusivement orale du savoir védique. Elle fait surtout intervenir, en dépit de l'écriture, une mémoire acoustique et non visuelle ${ }^{13}$. Par un autre contraste, il peut apparaître comme un paradoxe que la culture antique ayant le plus valorisé la parole publique, celle des Grecs, ait finalement donné par le biais de l'alphabet l'impulsion la plus dynamique au monde écrit. En Grèce ancienne, c'est la reproduction de la parole qui inaugure une production spécifique de l'écrit. Opérateur cognitif, l'écriture instaure des nouveaux régimes pour l'activité intellectuelle: si la première place revient à la loi écrite, la cartographie, la démonstration mathématique, les traités de médecine, les genres littéraires inédits que sont drame et philosophie en seront les dérivés les plus marquants ${ }^{14}$.

Les diverses écritures autorisent ainsi des formes de médiation relevant tout autant du geste rituel de l'échange, des langages spécifiquement lettrés, que de la parole quotidienne. Trois figures types du rapport verbal-écrit semblent hypothétiquement se dessiner: forte autonomie, favorisée par l'idéographie, de l'écrit par rapport à la parole, ou plurimédialité des deux niveaux; relative indépendance de la parole vis-à-vis de l'écriture, utilisée alors comme simple mémoire phonétique; enfin, interaction intense et remédiation de la parole par l'écriture, cas de l'alphabet valorisé comme véritable appareil cognitif. Nul doute que chaque type de structuration des modes d'inscription du langage renvoie, culturellement, à des formes distinctes d'orientation dans le monde. Une telle interrogation ne pourra donc se limiter aux seules techniques d'écriture. Elle devra nécessairement être complétée par la question: qui écrit, et dans quel but? En fin de compte, il faudrait comparer des ensembles de séries complexes au sein desquelles interfèrent la production des supports matériels (argile, papyrus, papier, etc.), le développement du médium technique lui-même (les types d'écritures), mais également les couches spécifiques de lettrés ou d'intellectuels qui sont ses porteurs attitrés, ainsi que le domaine de valorisation propre (religieux, économique, juridique, etc.) auquel le médium s'applique comme puissance d'autonomisation ${ }^{15}$. Alors

13. Voir Charles Malamoud, «Parole à voir et à entendre», dans Féminité de la parole: études sur l'Inde ancienne, Albin Michel, Paris, 2005, coll. «Sciences des religions", p. 61 sq., et Max Weber, Die Wirtschaftsethik der Weltreligionen II. Hinduismus und Buddhismus: Schriften 1916-1920 (MWS I/19), Tübingen, J.C.B. Mohr Siebeck, 1998, p. 116 sq. (Hindouisme et bouddhisme, trad. Isabelle Kalinowski, Paris, Éditions Flammarion, coll. «Champs», 2003, p. 282 sq.).

14. Marcel Détienne (dir.), Les savoirs de l'écriture en Grèce ancienne, Lille, Presses universitaires de Lille, 1992 [1988], p. 12 sq.

15. Voir entre autres Harold A. Innis, Empire and Communications, University of Toronto Press, 1972 [1950]. 


\begin{tabular}{|c|c|c|c|c|c|c|c|c|}
\hline & 090 & 091 & 092 & 093 & 094 & 095 & 096 & 097 \\
\hline & & ऐ & ठ & र & ओ & مّذ3 & ॠ & ○ \\
\hline & $\begin{array}{r}6 \\
0901\end{array}$ & ऑ & ड & र & $\mathrm{S}_{0.41}$ & $\begin{array}{r}1 \\
0951\end{array}$ & $\begin{array}{l}\text { ल्ह } \\
0951\end{array}$ & \\
\hline & $\begin{array}{r}\dot{0} \\
0902\end{array}$ & ओ & ढ & ल & $\underset{0942}{\bigcap}$ & $\frac{0}{0952}$ & $\overbrace{0962}$ & \\
\hline & $\begin{array}{l}0 \\
0903\end{array}$ & ओ & $\begin{array}{l}\text { ण } \\
0923\end{array}$ & $\bar{\Phi}$ & $\bigodot_{0.43}$ & 0 & ఝ & \\
\hline & $\begin{array}{l}\text { अ } \\
0904\end{array}$ & औ & त & क्ठ & है & 6 & 1 & \\
\hline & $\begin{array}{l}\text { अ } \\
0905\end{array}$ & क & थ & व & $\begin{array}{l}y \\
0.45\end{array}$ & & II & \\
\hline & $\begin{array}{l}\text { आ } \\
0006\end{array}$ & $\begin{array}{l}\text { ख } \\
0916\end{array}$ & द & $\begin{array}{l}\text { शा } \\
00956\end{array}$ & 0 & & 09566 & \\
\hline & $\begin{array}{c}\text { इ } \\
0907\end{array}$ & $\begin{array}{l}\text { ग } \\
0917\end{array}$ & धT & $\begin{array}{l}\bar{Q} \\
00937\end{array}$ & $\begin{array}{l}2 \\
0947\end{array}$ & & $?$ & \\
\hline & ${ }_{0908}$ & घ & न & $\begin{array}{l}\text { स } \\
\text { coss }\end{array}$ & 8 & क़ & $\sum_{0056}^{2}$ & \\
\hline 9 & उ & $\begin{array}{l}\text { ड. } \\
0919\end{array}$ & $\underset{0929}{7}$ & ह & † & ख़्र & ३ & \\
\hline & $\zeta_{000 A}$ & च & $\begin{array}{c}\text { प } \\
\text { 092A }\end{array}$ & & ठो & $\underset{\text { Ooss }}{\text { ग़ }}$ & $096 A$ & \\
\hline & $\begin{array}{l}\text { ॠ } \\
0908\end{array}$ & $\begin{array}{l}\bar{छ} \\
0918\end{array}$ & फ & & ो & $\begin{array}{l}\text { ज़ } \\
\text { oss }\end{array}$ & $\varphi$ & \\
\hline & $\begin{array}{l}\text { ल्ट } \\
090 c\end{array}$ & $\begin{array}{c}\text { ज } \\
091 c\end{array}$ & ब & $\underset{\cos c}{0}$ & $\begin{array}{c}\text { कौ } \\
\text { osac }\end{array}$ & ड़ & $\xi$ & \\
\hline & $\underset{0}{\tilde{J}}$ & $\begin{array}{l}\text { झ } \\
0910\end{array}$ & $\begin{array}{c}\text { H } \\
0920\end{array}$ & $\underset{0930}{S}$ & $\bigcap_{0.40}$ & 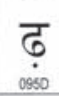 & $\underset{0050}{9}$ & $?$ \\
\hline & ऐ & ज & $\begin{array}{l}\text { म } \\
\text { O92E }\end{array}$ & $\begin{array}{c}\text { О } \\
093 E \\
0\end{array}$ & & फ़ & C & \\
\hline & $\sum_{090 F}^{5}$ & 己 & य & $\begin{array}{c}\text { fo } \\
\text { 09se }\end{array}$ & & $\begin{array}{l}\text { य } \\
\text { OSSE }\end{array}$ & $\rho_{O S B F}$ & \\
\hline
\end{tabular}

Fig. 2. "Devanagari», The Unicode Standard 4.1, (C) 1991-2005, p. 483. 
seulement la mesure dans laquelle l'écriture fait apparaître de nouveaux genres, et la question de leur signification, pourront être réévaluée.

\section{VI}

Ne faut-il pas également envisager, plus près de nous dans le temps, l'effet des machines à enregistrer sur le rapport langue-écriture? Parallèlement au travail de l'ethnographie, une grande partie de celui de la phonologie moderne a consisté à fixer par écrit les productions orales de cultures menacées. C'est en s'appuyant sur de nouveaux appareils, le phonographe puis le magnétophone, que l'ensemble (discret et fini) des variations phoniques de la totalité des langues parlées a pu 104 être enregistré, analysé et spécifiquement noté ${ }^{16}$. Système graphique à vocation « universelle», l'International Phonetic Alphabet (IPA), publié par l'Association phonétique internationale, affine le système d'oppositions sonores de l'alphabet traditionnel en fixant un signe (ou une combinaison de plusieurs) pour chaque sonorité distincte produite par l'articulation phonatoire. Cette notation à fonction avant tout scientifique augmente considérablement le nombre de signes graphiques, en particulier diacritiques, mais elle constitue de fait la remédiation la plus précise de la parole - en tant que faculté humaine - par l'écriture. Trace univoque de tous les sons articulés possibles, mémoire sonore des langues parlées, l'alphabet phonétique a pourtant eu un effet relativement limité, car détaché des échanges quotidiens.

Par voie de complément: de quel ordre est l'impact de l'ordinateur et de la communication électronique? Dès le télégraphe, la lettre a été codée de manière à pouvoir être transmise sous forme de signal électrique. L'encodage informatique ne procède pas de manière différente pour la transmission électronique des messages. Avec une norme de plus en plus exhaustive de codage de caractères comme Unicode, ce sont littéralement tous les systèmes d'écritures ayant cours à l'échelle de la planète qui se trouvent potentiellement réunis sur un même support. Il ne s'agit pas seulement d'écritures à référent purement linguistique, mais tout autant mathématique, musical et autres ensembles symboliques quelconques. De même, tous les types de logographie, écritures idéographiques, consonantiques, alphabétiques s'y côtoient. Parce que l'électricité comme médium se substitue au papier, tous les systèmes d'écritures sont rendus « homogènes » par le biais du calcul d'encodage (hexadécimal puis binaire) auquel ils sont indifférem-

16. Voir Claude Hagège, L'homme de paroles: contribution linguistique aux sciences humaines, Paris, Fayard, 1985, p. 94 sq. 
ment soumis. Avec l'encryptage et la diffusion électronique des messages, tout se passe comme si les débuts de la graphie linéaire dans la numération se répétaient à l'âge informatique : écrire au quotidien suppose désormais le calcul du codage. Est-il en ce sens plausible que la survie de telle ou telle langue passe aujourd'hui par son accession à une place dans ce code « universel »? Serait-ce alors le jeu de son calcul qui conditionnerait les formes de remédiation de la parole?

THE INTERNATIONAL PHONETIC ALPHABET (revised to 1993) CONSONANTS (PUIMONIC)

\begin{tabular}{|c|c|c|c|c|c|c|c|c|c|c|c|}
\hline & Bilabial & Labiodental & Dental & Aveolas & Patal veolas & \begin{tabular}{|l|l|l} 
\\
\end{tabular} & Palatal & Velas & Uvulat & Plaryugeal & Glotal \\
\hline Plosive & $\mathrm{p} \mathrm{b}$ & & & $t d$ & & $t d$ & c $\mathrm{f}$ & $\mathrm{kg}$ & q G & & $?$ \\
\hline Nasal & $\mathrm{m}$ & $\mathrm{m}$ & & $\mathrm{n}$ & & $\eta$ & $\mathrm{n}$ & 1] & $\mathrm{N}$ & & \\
\hline Trill & B & & & $r$ & & & & & R & & \\
\hline Tap of Flap & & & & r & & ᄃ & & & & & \\
\hline Fricative & $\phi \beta$ & f $v$ & $\theta$ б & S Z & $\int 3$ & $\$ \quad Z$ & ç $\dot{J}$ & $\mathrm{X} \quad \mathrm{Y}$ & $\chi$ в & h $\mathrm{F}$ & h f \\
\hline $\begin{array}{l}\text { Lateral } \\
\text { fricative }\end{array}$ & & & & 13 & & & & & & & \\
\hline Approximant & & v & & I & & $t$ & $\mathrm{j}$ & ul & & & \\
\hline $\begin{array}{l}\text { Lateral } \\
\text { approximant }\end{array}$ & & & & 1 & & l & $\Lambda$ & L & & & \\
\hline
\end{tabular}

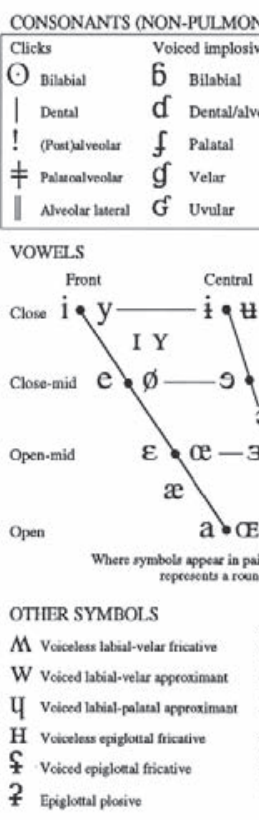

Ejectives
p' as in:
t' Dentabial
k' Velur
s' Alveolat fricative
SUPRASEGMENTALS

1. Primary stress founa'tIJen

TONES \& WORD ACCENT

1. Secondary stress "

LEVEL

WORD ACCENTS
CONTOU:

- Half-long $\mathrm{e}^{\prime}$

Eura-short

- Syllatile treak ri.akt

I Mince (foot) group

|| Major (istonatice) sroup

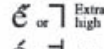

é $t_{\text {High }}$

e $\dashv$ mid

ě or $\Lambda$ Rising

è $f_{\text {Low }}$

è $」$ kxta

乙 Linkiag (abrence of a treak)

$\downarrow$ Dawnstap

ê $V_{\text {palling }}$

e $1_{\text {High rising }}$

è $\lambda_{\text {Low nixing }}$

$\uparrow$ Upstep

\begin{tabular}{|c|c|c|}
\hline DIACRITICS & \multicolumn{2}{|c|}{ Tiacrities may be placed atovere a symbel with a descender, e.2. 1) } \\
\hline Voiceless n & .. Breathy vicioed ba & ${ }_{n}$ Dental $\quad t \quad d$ \\
\hline $\mathrm{s} \mathrm{t}$ & $\sim$ Creaky voiced $\mathrm{b} \underset{\sim}{\mathrm{a}}$ & ـApical \\
\hline${ }^{h}$ Aspirated $t^{\text {h }} \mathrm{d}^{\mathrm{h}}$ & - Linguolabial $t \quad \mathrm{~d}$ & ¿ Laminal \\
\hline Moce rovended ? & ${ }^{\mathrm{w}}$ Lathializel $\mathrm{t}^{\mathrm{w}} \mathrm{d}^{\mathrm{w}}$ & $\sim$ Nasalized \\
\hline \& Leas roundeal ? & j Palealized & ${ }^{n}$ Nasal releste \\
\hline Advanced & ${ }^{\mathrm{Y}}$ velarized $\mathrm{t}^{\mathrm{Y}} \mathrm{d}^{\mathrm{Y}}$ & ${ }^{1}$ Lateral telcase $\mathrm{d}^{\mathrm{l}}$ \\
\hline Retracted & ${ }^{\mathrm{S}}$ Pharyngealized $\mathrm{t}^{\mathrm{S}} \mathrm{d}^{\mathrm{S}}$ & 'No autibish release $\mathrm{d}$ ' \\
\hline Centralized & $\sim$ Velarized $\propto$ p pharyagealize & 1 \\
\hline Mid-entralized & $e_{2}\left(\frac{I}{2}=\right.$ & voived alveolus fricative) \\
\hline , Syllabic & . Lowered & = vociced hilahial approximant) \\
\hline Non-syllabicie & Adrabeed Toegue Root & ę \\
\hline 2 Rhocicity & , Retracted Tangue Rood & ę \\
\hline
\end{tabular}

Fig. 3. Alphabet phonétique international (révisé en 1993). 


\begin{tabular}{|c|c|c|c|c|c|c|c|c|c|c|c|c|c|}
\hline 140 & 141 & 142 & 143 & 144 & 145 & 146 & 147 & 148 & 149 & $14 \mathrm{~A}$ & $14 \mathrm{~B}$ & $14 \mathrm{C}$ & $14 D$ \\
\hline & $\dot{\Delta}$ & 1 & $\Lambda$ & $\cdot>$ & $\supset$ & j. & $j$ & b & L & : & $\cdot \dot{\Gamma}$ & D & a \\
\hline & & & & & & & 1970 & & & 1440 & $\begin{array}{c}1480 \\
.\end{array}$ & 1400 & \\
\hline$\nabla$ & $\dot{\Delta}$ & $\smile$ & $\wedge$ & $>$. & 3 & $\cdot C$ & $\ddot{d}$ & $b^{\circ}$ & L & ८ & 广• & $\dot{\sigma}$ & $u$ \\
\hline 1401 & 1411 & 1421 & 1431 & 1441 & 1451 & 1451 & 1471 & 1481 & 1499 & 1441 & 1431 & $19 \mathrm{CI}$ & 1401 \\
\hline$\triangle$ & $\cdot D$ & n & 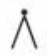 & $\dot{>}$ & $\ddot{\jmath}$ & C. & $b$ & 6 & \urcorner & $\mathrm{J}$ & • & $\sigma$ & م \\
\hline 1402 & 1412 & 1422 & 1432 & 1442 & 1452 & 1452 & 1472 & 1482 & 1492 & 140,2 & 1432 & 1402 & 1402 \\
\hline$\Delta$ & D. & ) & $>$ & $\dot{j}$ & $D$ & $\cdot \dot{C}$ & $\dot{b}$ & $b$ & 7. & 7 & 」. & $\dot{\sigma}$ & ד \\
\hline 1003 & 1413 & 1423 & 1433 & 1443 & 1453 & 1463 & 1473 & 1483 & 19993 & $14 \times 3$ & 1483 & ${ }_{14 C 3}$ & 1403 \\
\hline$\dot{\Lambda}$ & $\dot{\triangleright}$ & $\circ$ & $\dot{>}$ & $\cdot<$ & $\cdot$ & $\dot{c}$. & . & $d$ & • & 广ं & .j & 0 & $\stackrel{\circ}{\circ}$ \\
\hline 1004 & 1414 & 1624 & 1434 & 1444 & 1454 & 1454 & 1874 & 1684 & 1994 & 1404 & 1484 & $14 \mathrm{Ca}$ & 1404 \\
\hline$D$ & $\dot{D}$. & "I & $\ddot{y}$ & $<$. & $C$ & $: C$ & ৭. & $\mathrm{q}^{\prime}$ & r. & $\Gamma$ & $j$. & $\dot{0}$ & $\sigma$ \\
\hline 1005 & 1415 & 1425 & 1435 & 1445 & 1455 & 1455 & 1475 & 1405 & 1495 & 14,15 & 1485 & 1405 & 1405 \\
\hline$\dot{D}$ & $\dot{D}$ & 11 & D & $\dot{<}$ & ¿ & C & $\rho$ & $P^{\prime}$ & • & $\dot{\Gamma}$ &. & $\ddot{\cap}$ & $\dot{\subsetneq}$ \\
\hline 1406 & 1416 & 1426 & 1436 & 1446 & 1456 & 1465 & 1478 & 1486 & 1496 & 1406 & 1436 & ${ }_{1406}$ & 1405 \\
\hline$\ddot{\Delta}$ & $\bullet \triangleleft$ & - & $>$ & $\dot{<}$ & $\cdot U$ & $U^{\prime}$ & p. & $d^{\prime}$ & $\dot{\Gamma}$. & \rfloor & $L \cdot$ & 0 & حـ \\
\hline 1907 & 1417 & 1427 & 1437 & 1467 & 1457 & 1467 & 1477 & 1487 & 1497 & ${ }_{1447}$ & 1887 & 1067 & $\overline{1407}$ \\
\hline D) & $\triangleleft$ & - & $<$ & $\ddot{<}$ & U. & $\bigcap^{\prime}$ & $\cdot \dot{\rho}$ & $b^{\prime}$ & J & $j$ & . & $\dot{\mathrm{a}}$ & ز \\
\hline 1468 & 1418 & 1428 & 1438 & 1048 & 1458 & 1458 & 1478 & 1488 & 1498 & 1408 & 1488 & ${ }_{14 C B}$ & 1408 \\
\hline$\triangleright$ & $\cdot \dot{\triangleleft}$ & + & $\dot{<}$ & $<$ & $\cdot \bigcap$ & $\supset^{\prime}$ & p. & 7 & J. & $\ddot{\jmath}$ & L. & ס & ت \\
\hline 1009 & 1419 & 1429 & 1439 & 1449 & 1459 & 1469 & 1479 & 1489 & 1499 & 1649 & 1489 & 1409 & 1409 \\
\hline$\triangleleft$ & $\dot{\triangleleft} \cdot$ & $T$ & $\cdot V$ & 1 & ก. & $C^{\prime}$ & $\cdot d$ & ழீ & $j$ & $L$ & $: L$ & $\nabla^{*}$ & $C$ \\
\hline $140 x$ & $141 / A$ & 1924 & 1934 & $144 \mathrm{~A}$ & 145A & $146 \mathrm{~A}$ & $197 \mathrm{~A}$ & $18 B A$ & 149A & $16 A A$ & $18 B A$ & ${ }_{1 C C A}$ & $140 \mathrm{~A}$ \\
\hline$\dot{\Delta}$ & $\dot{\triangleleft}$ & $\nabla^{\prime}$ & $V$ & h & • & 9 & d. & $r$ & j. & L & L & ${ }^{\circ} \mathrm{a}$ & $\dot{C}$ \\
\hline 1408 & 1418 & 1428 & 1438 & 1448 & 1058 & 1468 & 1478 & 1488 & 1998 & TAAB & 1488 & ${ }_{14 C B}$ & ${ }_{1408}$ \\
\hline$\nabla$ & $w$ & $\Delta^{\prime}$ & $\wedge$ & $U$ & $\dot{n}$ & $\dot{\rho}$ & $\cdot \dot{j}$ & $\dot{\Gamma}$ & $\cdot L$ & $\cdot 7$ & $c$ & ه & כת \\
\hline $140 \mathrm{C}$ & IAIC & $162 \mathrm{C}$ & $143 \mathrm{C}$ & $144 \mathrm{C}$ & $145 \mathrm{C}$ & $148 \mathrm{C}$ & $147 \mathrm{C}$ & $149 \mathrm{C}$ & ${ }_{149 C}$ & IAAC & $148 \mathrm{C}$ & ${ }_{1 A C C}$ & $140 \mathrm{C}$ \\
\hline$\nabla \cdot$ & $\bullet^{\circ}$ & $D^{\prime}$ & $\wedge$ & 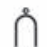 & .) & $P$ & j. & J & L. & 7 . & \lrcorner & ف & $\supset^{*}$ \\
\hline 1400 & 1410 & 1420 & 1430 & 1400 & 1450 & 1450 & 1470 & 1480 & 1490 & $14 A 0$ & 1880 & ${ }_{14 C D}$ & 1900 \\
\hline$\triangle$ & $\Delta$ & $\triangleleft$ & $\wedge$ & $\bigcap$ & ). & $\dot{\rho}$ & $b$ & j & ¿ & $\cdot\ulcorner$ & 2 & ¿ें & ${ }^{\circ} \mathrm{C}$ \\
\hline 1406 & 141E & $142 E$ & $143 E$ & 144E & 1458 & 166E & Ia7e & 14SE & IQSEE & 14AEE & $148 E$ & 14CE & $140 €$ \\
\hline$\triangle$ & , & V & $\AA$ & ก́ & ') & $d$ & $b^{\circ}$ & $\ddot{\jmath}$ & ¿. & $\Gamma$. & 2 & $\ddot{a}$ & $C$ \\
\hline$\overline{1006}$ & $1417 \mathrm{~F}$ & 1625 & 1435 & $144 \mathrm{~F}$ & 1455 & $1466 \mathrm{~F}$ & $147 \mathrm{~F}$ & $198 \mathrm{~B}$ & 1998 & 14AAF & 1495 & $14 C F$ & \\
\hline
\end{tabular}

Fig. 4. «Unified Canadian Aboriginal Syllabics », The Unicode Standard 4.1, (C) 1991-2005, p. 537. 